\title{
Switching stochastic models and applications in retrial queues
}

\author{
Vladimir V. Anisimov \\ Department of Industrial Engineering \\ Bilkent University, Bilkent 06533, Ankara, Turkey $\mathcal{G}$ \\ Department of Applied Statistics \\ Kiev University, Kiev 17, 252017, Ukraine \\ e-mail:vlanis@bilkent.edu.tr
}

\begin{abstract}
Some special classes of Switching Processes such as Recurrent Processes of a SemiMarkov type and Processes with Semi-Markov Switches are introduced. Limit theorems of Averaging Principle and Diffusion Approximation types are given. Applications to the asymptotic analysis of overloading state-dependent Markov and semi-Markov queueing models $M_{S M, Q} / M_{S M, Q} / 1 / \infty$ and retrial queueing systems $M / G / 1 / w . r$ in transient conditions are studied.
\end{abstract}

Key Words: Markov Process, Semi-Markov Process, Switching Process, Averaging Principle, Diffusion Approximation, Queueing Models, Retrial Queueing Systems

AMS subject classification: $60 \mathrm{~K} 25,60 \mathrm{~K} 15,60 \mathrm{~F} 17$

\section{Introduction}

Models of real information and computing systems have as usual a high dimension and a complex structure. Exact analytic solutions can be obtained only for special rare cases, and methods of a direct stochastic simulation work very slow and not efficient. Therefore asymptotic methods play the basic role at the investigation and approximate modelling.

In the paper a new approach is suggested for study an asymptotic behavior of wide classes of overloading queueing models and in particular retrial queueing systems in transient and stable regimes. This approach is based on limit theorems of Averaging Principle $(A P)$ and Diffusion Approximation $(D A)$ types for so called Switching Processes $(S P)$.

The paper was supported by INTAS Project 96-0828

Received: September 22, 1998; Accepted: December 4, 1998 
$S P$ have a property that the character of their development varies spontaneously at some epochs of time which can be random functionals of the previous trajectory.

The class of $S P$ was introduced by Anisimov $(1975,1977) . S P$ are the natural generalization of such well-known classes of random processes as Markov processes homogeneous in the 2nd component (Ežov and Skorokhod, 1969), processes with independent increments and semi-Markov switches (Anisimov, 1973), piecewise Markov aggregates (Buslenko et. al., 1973), Markov processes with semi-Markov interference of chance (Gikhman and Skorokhod, 1973) and Markov and semi-Markov evolutions (Papanicolaou and Hersh, 1972; Hersh, 1974; Kertz, 1978; Kurtz, 1973; Pinsky, 1975ab; Korolyuk and Swishchuk, 1994).

Theorems, concerning the convergence of the trajectory of $S P$ to a solution of some ordinary differential equation $(A P)$ and the convergence of the normed difference to some diffusion process $(D A)$, were proved by Anisimov $(1989,1991,1992,1994,1995 \mathrm{ab})$ and Anisimov and Aliev (1990) for different important subclasses of $S P$ : recurrent processes of a semi-Markov type (RPSM), processes with semi-Markov switches and general $S P$ with feedback between both components. The results of $A P$ type for stochastic differential equations (Khas'minskii, 1968) and for some Markov models in the case of feedback (Skorokhod, 1987) based on a martingale technique also are very closely connected with this direction.

Different asymptotic approaches for various classes of general queueing systems are considered in books of Buslenko et al.(1973), Basharin et al. (1989), Anisimov et al. (1987), and papers of Chen and Mandelbaum (1994), Harrison (1995), Harrison and Williams (1996), Anisimov (1998), Mandelbaum and Pats (1998), (see also references there). A new approach based on $A P$ and $D A$ type theorems for $S P$ was used by Anisimov (1989, 1995a,b, 1996) and in the book of Anisimov and Lebedev (1992) for study various classes of state-dependent Markov queueing systems and networks in conditions of heavy loading. For non-Markov models some results were obtained by Anisimov (1989, 1995ab, 1996).

Retrial queues is comparatively a new direction in queueing models. Over recent years there were appeared many publications concerning the developing of approximating methods and analysis of steady-state behaviour for different classes of retrial queueing models (see reviews of Yang and Templeton (1987), Falin (1990) and Kulkarni and Liang (1997), papers 
of Artalejo (1995), Artalejo and Falin $(1995,1996)$, Martin and Artalejo (1995), and book of Falin and Templeton (1997).

The 4th section of the paper is devoted to applications of $A P$ and $D A$ type theorems for $S P$ in the asymptotic analysis of some classes of overloading state-dependent Markov and semi-Markov queueing models as well as for retrial queueing systems in transient conditions. Some results in this direction were obtained by Anisimov and Atadzhanov $(1991,1994)$ and Anisimov (1999).

\section{Switching Stochastic Processes}

$S P$ can be described as two-component processes $(x(t), \zeta(t)), t \geq 0$, with the property existing a sequence of epochs $t_{1}<t_{2}<\cdots$ such that on each interval $\left[t_{k}, t_{k+1}\right), x(t)=x\left(t_{k}\right)$ and the behaviour of the process $\zeta(t)$ depends on the value $\left(x\left(t_{k}\right), \zeta\left(t_{k}\right)\right)$ only. Epochs $t_{k}$ are switching times and $x(t)$ is the discrete switching component. A general definition of $S P$ was given by Anisimov $(1975,1977)$. Now we consider some important subclasses of $S P$ which appear in different applications.

\subsection{Recurrent processes of a semi-Markov type (RPSM)}

Let $F_{k}=\left\{\left(\xi_{k}(\alpha), \tau_{k}(\alpha)\right), \alpha \in \mathcal{R}^{r}\right\}, k \geq 0$, be jointly independent families of random variables with values in $\mathcal{R}^{r} \times[0, \infty)$ and $S_{0}$ be independent of $F_{k}, k \geq 0$ random variable in $\mathcal{R}^{r}$. We assume here (and further) that variables introduced are measurable in the ordinary way in the variable $a$ concerning $\sigma$-algebra $B_{\mathcal{R}^{r}}$. Denote $t_{0}=0, t_{k+1}=t_{k}+\tau_{k}\left(S_{k}\right), S_{k+1}=$ $S_{k}+\xi_{k}\left(S_{k}\right), k \geq 0$ and put

$$
S(t)=S_{k} \text { as } t_{k} \leq t<t_{k+1}, t \geq 0 .
$$

Then a process $S(t)$ forms a simple recurrent process of a semi-Markov type (RPSM) (see Anisimov, 1989, Anisimov and Aliev, 1990).

In the case when distributions of variables $\left(\xi_{k}(\alpha), \tau_{k}(\alpha)\right)$ do not depend on the parameter $k$, the process $S(t)$ is a homogeneous semi-Markov process $(S M P)$. If in addition these distributions do not depend on the parameter $\alpha$, epochs $t_{k}, k \geq 0$ form a recurrent flow and $S(t)$ is a generalized renewal 
process. In particular when variables $\tau_{k}(\alpha)$ have exponential distributions, a process $S(t)$ is a Markov process $(M P)$.

Further let $\tilde{F}_{k}=\left\{\left(\xi_{k}(x, \alpha), \tau_{k}(x, \alpha)\right), x \in X, \alpha \in \mathcal{R}^{r}\right\}, k \geq 0$ be jointly independent families of random variables with values in $\mathcal{R}^{r} \times[0, \infty)$, and let $x_{l}, l \geq 0$ be an independent of $\tilde{F}_{k}, k \geq 0 M P$ with values in $X,\left(x_{0}, S_{0}\right)$ be an initial value. We put $t_{0}=0, t_{k+1}=t_{k}+\tau_{k}\left(x_{k}, S_{k}\right), S_{k+1}=$ $S_{k}+\xi_{k}\left(x_{k}, S_{k}\right), k \geq 0$, and denote

$$
S(t)=S_{k}, x(t)=x_{k} \text { as } t_{k} \leq t<t_{k+1}, t \geq 0 .
$$

Then the process $(x(t), S(t))$ forms a $R P S M$ with Markov switches. When distributions of variables $\tau_{k}(x, \alpha)$ do not depend on parameters $\alpha$ and $k$ the process $x(t)$ is a semi-Markov process (SMP).

\subsection{Processes with semi-Markov switches (PSMS)}

Now we consider in some sense more general construction when we have an evolution of some random process in a semi-Markov environment.

Let $F_{k}=\left\{\zeta_{k}(t, x, \alpha), t \geq 0, x \in X, \alpha \in \mathcal{R}^{r}\right\}, \quad k \geq 0$ be jointly independent families of random processes, where $\zeta_{k}(t, x, \alpha)$ at each fixed $k, x, \alpha$ be a process with trajectories in Skorokhod space $\mathcal{D}_{\infty}^{r}, x(t), t \geq 0$ be an independent of $F_{k}, k \geq 0 S M P$ in $X$ and $S_{0}$ be an initial value. Denote by $0=t_{0}<t_{1}<\cdots$ the epochs of sequential jumps for $x(\cdot)$ and put $x_{k}=x\left(t_{k}\right), k \geq 0$. We construct a process with semi-Markov switches (or in a semi-Markov environment) in the following way: put $S_{k+1}=S_{k}+\xi_{k}$, where $\xi_{k}=\zeta_{k}\left(\tau_{k}, x_{k}, S_{k}\right), \tau_{k}=t_{k+1}-t_{k}$, and denote

$$
\zeta(t)=S_{k}+\zeta_{k}\left(t-t_{k}, x_{k}, S_{k}\right) \text { as } t_{k} \leq t<t_{k+1}, t \geq 0 .
$$

Then a two-component process $(x(t), \zeta(t)), t \geq 0$ is the process with semi-Markov switches (PSMS). If we introduce also an imbedded process $S(t)=S_{k}$ as $t_{k} \leq t<t_{k+1}, t \geq 0$, then the process $(x(t), S(t))$ forms a RPSM with Markov switches.

In the case when $\{\zeta(t, x), t \geq 0\}$ is the family of Markov processes and $\zeta(t, x, \alpha)$ denotes the process $\zeta(t, x)$ with initial value $\alpha$, we get that the process $(x(t), \zeta(t))$ forms a Markov random evolution (when the process $x(t)$ is a $M P$ ) or semi-Markov evolution (when the process $x(t)$ is a $S M P$ ) 
(see Hersh, 1974; Kurtz, 1973; Kertz, 1978; Pinsky, 1975; Korolyuk and Swishchuk, 1994).

In particular if $\{\zeta(t, x), t \geq 0\}$ is the family of processes with independent increments and the process $x(t)$ is a $M P$, then the pair $(x(t), \zeta(t)), t \geq 0$ forms a $M P$ homogeneous in the 2nd component (see Ežov and Skorokhod, 1969). If $x(t)$ is a $S M P$ then the pair $(x(t), \zeta(t)), t \geq 0$ forms a process with independent increments and semi-Markov switches (see Anisimov, 1973).

In terms of $S P$ it is possible to describe various classes of stochastic models such as state-dependent queueing systems and networks of the types $S M_{Q} / M_{Q} / 1 / \infty, M_{S M, Q} / M_{S M, Q} / l / k,\left(M_{S M, Q} / M_{S M, Q} / l_{i} / k_{i}\right)^{r}$ with batch Markov or semi-Markov input, finite number of nodes, different types of calls (impatient calls and possibly of a random size - volume of information or necessary job) and batch state-dependent service, switched by some external semi-Markov environment and current values of queues.

Example 2.1. Stochastic network $\left(M_{S M, Q} / M_{S M, Q} / 1 / \infty\right)^{r}$.

We consider an information network consisting of $r$ nodes with one server in each node. Suppose for simplicity that each node has an infinite capacity. Let $S M P x(t)$ with values in some space $X$, families of nonnegative functions $\left\{\lambda(x, \bar{q}), \mu_{i}(x, \bar{q}), i=\overline{1}, r, x \in X, \bar{q} \in R_{+}^{r}\right\}$, independent families of random vectors $\left\{\bar{\eta}(x, \bar{q}), x \in X, \bar{q} \in R_{+}^{r}\right\}$ and $\left\{\bar{\kappa}_{i}(x, \bar{q}), i=\overline{1, r}, x \in\right.$ $\left.X, \bar{q} \in R_{+}^{r}\right\}$ with values in $R_{+}^{r}$ and $R_{+}^{r+1}$ respectively be given. Denote by $Q_{i}(t)$ the total volume of the information in the $i$-th node and put $\bar{Q}(t)=\left(Q_{1}(t), \ldots, Q_{r}(t)\right)$.

We assume that if $x(t)=x, \bar{Q}(t)=\bar{q}=\left(q_{1}, \ldots, q_{\tau}\right)$, then with intensity $\lambda(x, \bar{q})$ a call of a random size $\bar{\eta}(x, \bar{q})$ may enter the system (it means that the $i$-th component of the vector $\bar{\eta}(x, \bar{q})$ enters the $i$-th node). Correspondingly if $x(t)=x, \bar{Q}(t)=\bar{q}$, then with intensity of service $\mu_{i}(x, \bar{q})$ the random portion of the information of the size $\kappa_{i}(x, \bar{q})$ may leave the $i$-th node, where $\kappa_{i}(x, \bar{q})=\sum_{j=0}^{r} \kappa_{i}^{(j)}(x, \bar{q})$, and $\kappa_{i}^{(j)}(x, \bar{q})$ is $j$-th component of the vector $\bar{\kappa}_{i}(x, \bar{q})$. This means that the value $\kappa_{i}^{(j)}(x, \bar{q})$ passes into the $j$-th node, $j=\widetilde{1, r}$, and the portion $\kappa_{i}^{(0)}(x, \bar{q})$ leaves the network (we can always assume that $\left.\kappa_{i}(x, \bar{q}) \leq q_{i}\right)$.

To describe the process $(x(t), \bar{Q}(t))$ in the network as a $S P$, we introduce independent families of multi-dimensional Markov processes $\left\{\bar{\gamma}_{k}(t, x, \bar{q}), t \geq\right.$ $\left.0, x \in X, \bar{q} \in R_{+}^{r}\right\}, k \geq 0$ with values in $R_{+}^{r}$ in the following way: $\bar{\gamma}_{k}(0, x, \bar{q})=$ 
$\bar{q}$ and if $\bar{\gamma}_{k}(t, x, \bar{q})=\bar{s}$ then with intensity $\Lambda(x, \bar{s})=\lambda(x, \bar{s})+\sum_{i=1}^{r} \mu_{i}(x, \bar{s})$ the process can get a jump of the size $\bar{\beta}(x, \bar{s})$ where

$$
\bar{\beta}(x, \bar{s})= \begin{cases}\bar{\eta}(x, \bar{s}) & \text { with prob. } \lambda(x, \bar{s}) \Lambda(x, \bar{s})^{-1} \\ -\kappa_{i}(x, \bar{s}) \bar{e}_{i}+\left[\kappa_{i}(x, \bar{s})\right]_{0} & \text { with prob. } \mu^{(i)}(x, \bar{s}) \Lambda(x, \bar{s})^{-1} \\ & i=\overline{1, r}\end{cases}
$$

where we denote for the vector $\bar{b}=\left(b^{(i)}, i=\overline{0, r}\right)$ by $[\bar{b}]_{0}$ the vector $\left(b^{(i)}, i=\right.$ $\overline{1, r})$, and $\bar{e}_{i}$ is the vector with $i$-th component equal 1 and others equal 0 .

Now we introduce the family of processes $\zeta_{k}(t, x, \alpha)$ in the following way: $\zeta_{k}(t, x, \alpha)=\gamma_{k}(t, x, \alpha)-\alpha, t \leq \tau_{k}(x)$.

Then the process $(x(t), Q(t))$ constructed by introduced families $\zeta_{k}(\cdot)$ and $S M P x(\cdot)$ (see (2.3)) is the process with semi-Markov switches.

By analogy we may also describe the networks $\left(S M / M_{S M, Q} / 1 / \infty\right)^{r}$ with semi-Markov input (calls enter at epochs of jumps of $x(\cdot)$ ).

\section{Averaging principle and diffusion approximation for PSMS}

Consider a triangular scheme. Let for each $n>0 \quad F_{n k}=\left\{\zeta_{n k}(t, x, \alpha), t \geq\right.$ $\left.0, x \in X, \alpha \in R^{r}\right\}, \quad k \geq 0$ be jointly independent families of random processes in $D_{\infty}^{r}, x_{n}(t), t \geq 0$ be an independent of them $S M P$ in $X, S_{n 0}$ be an initial value. Let also $0=t_{n 0}<t_{n 1}<\cdots$ be the epochs of sequential jumps of $x_{n}(\cdot), x_{n k}=x_{n}\left(t_{n k}\right), k \geq 0$. We construct a $P S M S$ according to formulae (2.3): put $S_{n k+1}=S_{n k}+\xi_{n k}$, where $\xi_{n k}=\zeta_{n k}\left(\tau_{n k}, x_{n k}, S_{n k}\right), \tau_{n k}=t_{n k+1}-t_{n k}$, and denote

$$
\zeta_{n}(t)=S_{n k}+\zeta_{n k}\left(t-t_{n k}, x_{n k}, S_{n k}\right) \text { as } t_{n k} \leq t<t_{n k+1}, t \geq 0 .
$$

Then the process $\left(x_{n}(t), \zeta_{n}(t)\right), t \geq 0$ is a PSMS.

At first we study an $A P$ for the switched component $\zeta_{n}(\cdot)$. Consider for simplicity a homogeneous case (distributions of processes $\zeta_{n k}(\cdot)$ do not depend on the index $k \geq 0$ ). Let $\tau_{n}(x)$ be a sojourn time in the state $x$ for $S M P x_{n}(\cdot)$. Denote for each $x \in X, \alpha \in R^{r}$

$$
\xi_{n}(x, \alpha)=\zeta_{n 1}\left(\tau_{n}(x), x, \alpha\right), g_{n}(x, \alpha)=\sup _{t<\tau_{n}(x)}\left|\zeta_{n 1}(t, x, \alpha)\right|
$$


Suppose that $M P x_{n k}, k \geq 0$ has at each $n \geq 0$ a stationary measure $\pi_{n}(A), A \in B_{X}$ and denote $m_{n}(x)=\mathbf{E} \tau_{n 1}(x), b_{n}(x, \alpha)=\mathbf{E} \xi_{n 1}(x, n \alpha)$,

$$
m_{n}=\int_{X} m_{n}(x) \pi_{n}(\mathrm{~d} x), \quad b_{n}(\alpha)=\int_{X} b_{n}(x, \alpha) \pi_{n}(\mathrm{~d} x),
$$

$\alpha_{n}(k)=\sup _{A, B \in B_{X}, i \geq 0}\left|\mathbf{P}\left\{x_{n i} \in A, x_{n i+k} \in B\right\}-\mathbf{P}\left\{x_{n i} \in A\right\} \mathbf{P}\left\{x_{n i+k} \in B\right\}\right|$.

Theorem 3.1. (Averaging principle). Suppose that $n^{-1} S_{n o} \stackrel{p}{\rightarrow} s_{0}$ and:

1) there exists a sequence of integers $r_{n}$ such that $n^{-1} r_{n} \rightarrow O$ and $\sup _{k \geq r_{n}} \alpha_{n}(k) \rightarrow O$;

2) for any $N>0, \varepsilon>0 \quad \lim _{n \rightarrow \infty} \sup _{x,|\alpha|<N} \mathbf{P}\left\{n^{-1} g_{n}(x, \alpha)>\epsilon\right\}=0$;

3) $\lim _{L \rightarrow \infty} \lim \sup _{n \rightarrow \infty} \sup _{|\alpha|<N} \sup _{x}\left\{\mathbf{E} \tau_{n 1}(x) \chi\left(\tau_{n 1}(x)>L\right)+\right.$ $\left.\mathbf{E}\left|\xi_{n 1}(x, n \alpha)\right| \chi(|\xi(x, n \alpha)|>L)\right\}=0$;

4) for any $x$ as $\max \left(\left|\alpha_{1}\right|,\left|\alpha_{2}\right|\right)<N$ $\left|b_{n}\left(x, \alpha_{1}\right)-b_{n}\left(x, \alpha_{2}\right)\right|<C_{N}\left|\alpha_{1}-\alpha_{2}\right|+\alpha_{n}(N)$, where $C_{N}$ are some constants, $\alpha_{n}(N) \rightarrow 0$ uniformly on $\left|\alpha_{1}\right|<N,\left|\alpha_{2}\right|<$ $N$;

5) there exists a function $b(\alpha)$ and a constant $m$ such that for any $\alpha \in R^{r} \quad b_{n}(\alpha) \rightarrow b(\alpha), m_{n} \rightarrow m>0$.

Then for any $T>0$

$$
\sup _{0 \leq t \leq T}\left|n^{-1} \zeta_{n}(n t)-s(t)\right| \stackrel{p}{\rightarrow} 0
$$

where

$$
s(0)=s_{0}, \quad \mathrm{~d} s(t)=m^{-1} b(s(t)) \mathrm{d} t
$$

(it is supposed that a solution of the equation exists on each interval and is unique).

Remark 3.1. Condition 1) covers also more general situations than only the case when the process $x_{n k}$ is ergodic in the limit. For instance state space can form an $S$-set (see Anisimov, 1973, 1996).

Now let us consider a $D A$ for the sequence of processes $\theta_{n}(t)=n^{-1 / 2}\left(\zeta_{n}(n t)-n s(t)\right)$. 
We introduce a uniformly strong mixing coefficient: $\varphi_{n}(r)=\sup _{x, y, A}\left|\mathbf{P}\left\{x_{n r} \in A / x_{n o}=x\right\}-P\left\{x_{n r} \in A / x_{n o}=y\right\}\right|$.

Put $\quad \tilde{b_{n}}(\alpha)=b_{n}(\alpha) m_{n}^{-1}, \quad \tilde{b}(\alpha)=b(\alpha) m^{-1}, \quad \rho_{n k}(x, \alpha)=\xi_{n k}(x, n \alpha)-$ $b_{n}(x, \alpha)-\tilde{b}(\alpha)\left(\tau_{n k}(x)-m_{n}(x)\right), \quad D_{n}(x, \alpha)^{2}=E \rho_{n 1}(x, \alpha) \rho_{n 1}(x, \alpha)^{*}$, $\gamma_{n}(x, \alpha)=b_{n}(x, \alpha)-b_{n}(\alpha)-\tilde{b}(\alpha)\left(m_{n}(x)-m_{n}\right)$.

Theorem 3.2. (Diffusion Approximation). Suppose that $\theta_{n}(0) \stackrel{\mathrm{W}}{\Rightarrow} \theta_{0}$, there exist fixed $r>0$ and $q \in[0,1)$ such that $\varphi_{n}(r) \leq q, n>0$, conditions of Theorem 3.1 hold where $\sqrt{n} \alpha_{n}(N) \rightarrow 0$, and for any $N>0$ :

1) $\lim _{n \rightarrow \infty} \sup _{|\alpha|<N} \sup _{x} n \mathbf{P}\left\{n^{-1 / 2} g_{n}(x, \alpha)>\varepsilon\right\}=0, \forall \varepsilon>0$;

2) $\lim _{L \rightarrow \infty} \lim \sup _{n \rightarrow \infty} \sup _{|\alpha|<N} \sup _{x}\left\{\mathbf{E} \tau_{n 1}(x)^{2} \chi\left(\tau_{n 1}(x)>L\right)+\right.$ $\left.\mathbf{E}\left|\xi_{n 1}(x, n \alpha)\right|^{2} \chi\left(\left|\xi_{n 1}(x, n \alpha)\right|>L\right)\right\}=0$;

3) $\left|D_{n}\left(x, \alpha_{1}\right)^{2}-D_{n}\left(x, \alpha_{2}\right)^{2}\right| \leq C_{N}\left|\alpha_{1}-\alpha_{2}\right|+\alpha_{n}(N)$, as $\max \left(\left|\alpha_{1}\right|,\left|\alpha_{2}\right|\right)<$ $N$, where $\alpha_{n}(N) \rightarrow 0$ uniformly in $\left|\alpha_{1}\right|<N,\left|\alpha_{2}\right|<N$;

4) there exists a function $q(\alpha, z)$ such that for any $N$ in the domain $|\alpha|<N \quad|q(\alpha, z)|<C_{N}(1+|z|)$ and uniformly in $|\alpha|<N$ at each fixed $z \quad \sqrt{n}\left(\tilde{b}_{n}\left(\alpha+n^{-1 / 2} z\right)-\tilde{b}(\alpha)\right) \rightarrow q(\alpha, z) ;$

5) there exist functions $D(\alpha)$ and $B(\alpha)$ such that for any $\alpha \in R^{m}$ $D_{n}(\alpha)^{2}=\int_{X} D_{n}(x, \alpha) \pi_{n}(\mathrm{~d} x) \rightarrow D(\alpha)^{2}, B_{n}^{(1)}(\alpha)^{2}+B_{n}^{(2)}(\alpha)^{2}+\left(B_{n}^{(2)}(\alpha)^{*}\right)^{2} \rightarrow$ $B(\alpha)^{2}$, where $B_{n}^{(1)}(\alpha)^{2}=\int_{X} \gamma_{n}(x, \alpha) \gamma_{n}(x, \alpha)^{*} \pi_{n}(\mathrm{~d} x), B_{n}^{(2)}(\alpha)^{2}=$ $\sum_{k \geq 1} \mathbf{E} \gamma_{n}\left(x_{n o}, \alpha\right) \gamma_{n}\left(x_{n k}, \alpha\right)^{*}$, at $\mathbf{P}\left\{x_{n o} \in A\right\}=\pi_{n}(A), A \in B_{X}$.

Then the sequence of processes $\theta_{n}(t)$ weakly converges at each $T>0$ in the space $\mathcal{D}_{T}^{r}$ to the diffusion process $\theta(t)$ :

$$
\theta(0)=\theta_{0}, \mathrm{~d} \theta(t)=q(s(t), \theta(t)) \mathrm{d} t+m^{-1 / 2}\left(D(s(t))^{2}+B(s(t))^{2}\right)^{1 / 2} \mathrm{~d} w(t),
$$

where $w(t)$ is a standard Wiener process in $R^{r}$, and the solution of this equation exists and is unique.

The proof of these theorems can be found in Anisimov, 1994. These results also can be extended on nonhomogeneous in time models (see Anisimov, 1995). 


\section{Diffusion approximation in queueing models}

\subsection{Markov models}

We consider a general Markov queueing system of the type $\bar{M}_{\bar{Q}} / \bar{M}_{\bar{Q}} / 1 / \infty$ which includes state-dependent systems with group input and service, systems with impatient calls, and even Markov networks.

Suppose that characteristics of the system depends on a parameter $n, n \rightarrow \infty$. Let non-negative functions $\lambda(q), \mu(q), \nu_{i}(q), i=\overline{1, m}, q \in R_{+}^{m}$ be given where $R_{+}^{m}=[0, \infty)^{m}$. Let also $\alpha(q), \gamma(q), \beta_{i}(q), i=\overline{1, m}, q \in R_{+}^{m}$ be random variables with values in $R_{+}^{m}$. The system consists of one server and infinitely many places for waiting. Denote by $Q_{n}(t)$ the number of calls in the system at time $t, Q_{n}(t) \in R_{+}^{m}$. Vector values may denote the different types of calls or different priorities. The system is working in the following way: if $Q_{n}(t)=n q$, then with intensity $\lambda(q) \alpha(q)$ calls may enter the system, and correspondingly with intensity of service $\mu(q) \gamma(q)$ calls may finish service. In addition to this each call of the type $i$ independently of others with intensity $n^{-1} \nu_{i}(q)$ may transform into $e_{i}+\beta_{i}(q)$ calls, where $e_{i}$ is a vector with $i$-th component is equal one and other components are equal 0 . Vector $\beta_{i}(q)$ may have positive and negative elements. All calls that enter the system, stand in the queue, calls after finishing service leave the system.

By the construction the process $Q_{n}(t)$ is a multidimensional $M P$. We represent it now as RPSM.

Denote by $t_{n k}, k \geq 0$ epochs of sequential jumps of $Q_{n}(t)$ and put $\tau_{n k}=$ $t_{n k+1}-t_{n k}, Q_{n k}=Q_{n}\left(t_{n k}+0\right), \xi_{n k}=Q_{n k+1}-Q_{n k}, k \geq 0$. Let us introduce jointly independent families of random variables $\tau_{n k}(n q)$ and $\xi_{n k}(n q)$ such that $\mathbf{P}\left\{\tau_{n k}(n q)<t\right\}=P\left\{\tau_{n k}<t / Q_{n k}=n q\right\}, \quad \mathbf{P}\left\{\xi_{n k}(n q) \in A\right\}=$ $P\left\{\xi_{n k} \in A / Q_{n k}=n q\right\}$.

In terms of introduced variables the process $Q_{n}(t)$ is a $R P S M$. In our case it can be easily seen that the value $\tau_{n k}(n q)$ has an exponential distribution with parameter $\Lambda(q)=\lambda(q)+\mu(q)+\nu(q)$ where $\nu(q)=\sum_{i=1}^{m} q_{i} \nu_{i}(q)$, $q=\left(q_{1}, \ldots, q_{m}\right)$, and the value $\xi_{n k}(n q)$ can be represented in the form:

$$
\xi_{n 1}(n q)=\left\{\begin{array}{lll}
\alpha(q) & \text { with probability } & \lambda(q) \Lambda(q)^{-1} \\
-\gamma(q) & \text { with probability } & \mu_{i}(q) \Lambda(q)^{-1} \\
\beta_{i}(q) & \text { with probability } & q_{i} \nu_{i}(q) \Lambda(q)^{-1}, i=\overline{1, m}
\end{array}\right.
$$

According to the construction the trajectory of RPSM coincides with tra- 
jectory $Q_{n}(t)$ on some interval $[0, T]$ only if its trajectory on this interval completely belongs to the space $R_{+}^{m}$.

Let us introduce the following values: $m^{(1)}(q)=\mathbf{E} \alpha(q), m^{(2)}(q)=$ $\mathbf{E} \gamma(q), m_{i}^{(3)}(q)=\mathbf{E} \beta_{i}(q), d^{(1)}(q)=\mathbf{E} \alpha(q) \alpha(q)^{*}, d^{(2)}(q)=\mathbf{E} \gamma(q) \gamma(q)^{*}$, $d_{i}^{(3)}(q)=\mathbf{E} \beta_{i}(q) \beta_{i}(q)^{*}$, and denote:

$b(q)=m^{(1)}(q) \lambda(q)-m^{(2)}(q) \mu(q)+\sum_{i=1}^{m} m_{i}^{(3)}(q) q_{i} \nu_{i}(q)$, $B^{2}(q)=d^{(1)}(q) \lambda(q)+d^{(2)}(q) \mu(q)+\sum_{i=1}^{m} d_{i}^{(3)}(q) q_{i} \nu_{i}(q)$.

Let also $G(q)$ be the matrix of partial derivatives for $b(q)$ that is $\lim _{h \rightarrow 0} h^{-1}(b(q+h z)-b(q))=G(q) z, z \in R^{m}$.

Theorem 4.1. I) Let the variables $\alpha(q), \gamma(q), \beta(q)$ be uniformly in $q$ integrable in each bounded region in $R_{+}^{m}$, functions $\lambda(q), \mu(q), \nu_{i}(q), m_{i}^{(j)}(q)$ be locally Lipschitz, $\Lambda(q)>0, q \in R_{+}^{m}$ and $n^{-1} Q_{n}(0) \stackrel{\mathrm{P}}{\longrightarrow} s_{0}$.

Then

$$
\sup _{0 \leq t \leq T}\left|n^{-1} Q_{n}(n t)-s(t)\right| \stackrel{\mathrm{P}}{\longrightarrow} 0,
$$

the function $s(t)$ satisfies the equation: $\mathrm{d} s(t)=b(s(t)) \mathrm{d} t, \dot{s}(0)=s_{0}$, and $T$ is any positive value such that $y(+\infty)>T$ and in each component $s(t) \geq 0$ as $t \leq T$, where $y(t)=\int_{0}^{t} q(\eta(u))^{-1} \mathrm{~d} u$, and the function $\eta(t)$ satisfies the equation $\eta(0)=s_{0}, \mathrm{~d} \eta(t)=b(\eta(t)) q(\eta(t))^{-1} \mathrm{~d} t$

(it is assumed that the solution of this equation exists and is unique).

2). Suppose that in addition to these conditions variables $|\alpha(q)|^{2},|\gamma(q)|^{2}$, $|\beta(q)|^{2}$ are integrable uniformly in $q$ in each bounded region in $R_{+}^{m}$, functions $B(q)$ and $G(q)$ are continuous and $n^{-1 / 2}\left(Q_{n}(0)-n s_{0}\right) \stackrel{\text { w }}{\Rightarrow} \zeta_{0}$.

Then the sequence of processes $\zeta_{n}(t)=n^{-1 / 2}\left(Q_{n}(n t)-n s(t)\right)$ weakly converges in $\mathcal{D}_{T}^{m}$ to a diffusion process $\zeta(t)$ satisfying the equation:

$$
\mathrm{d} \zeta(t)=G(s(t)) \zeta(t) \mathrm{d} t+B(s(t)) \mathrm{d} w(t), \zeta(0)=\zeta_{0} .
$$

Proof. In our case the process $Q_{n}(t)$ is represented as a simple $R P S M$ and the proof directly follows from Theorems 3.1, 3.2 given in Anisimov (1995a) (see also Anisimov, 1995b).

Example 4.1. Consider state-dependent system $M_{Q} / M_{Q} / 1 / \infty$ with impatient calls. It means that if $Q_{n}(t)=n q$, then the local intensities of input 
and service are correspondingly $\lambda(q)$ and $\mu(q)$, and each call in the queue independently of others with intensity $n^{-1} \nu(q)$ may leave the system. Here we suppose for simplicity that $\alpha(\cdot) \equiv 1, \gamma(\cdot) \equiv 1$.

Then in notations of Theorem $4.1 \beta(a)=-1, \Lambda(a)=\lambda(a)+\mu(a)+$ $a \nu(a), b(a)=\lambda(a)-\mu(a)-a \nu(a), B^{2}(a)=\lambda(a)+\mu(a)+a \nu(a), G(a)=$ $\lambda^{\prime}(a)-\mu^{\prime}(a)-\nu(a)-a \nu^{\prime}(a)$, and we can write corresponding equations for $s(t)$ and $\zeta(t)$.

In particular if $\lambda(q) \equiv \lambda, \mu(q) \equiv \mu, \nu(q) \equiv \nu$, we get: $\mathrm{d} s(t)=(\lambda-\mu-$ $\nu s(t)) \mathrm{d} t, s(0)=s_{0}$ and $\mathrm{d} \zeta(t)=-\nu \zeta(t) \mathrm{d} t+(\lambda+\mu+\nu s(t))^{1 / 2} \mathrm{~d} w(t)$.

Solving these equations we find:

$$
s(t)=\nu^{-1}(\lambda-\mu)-\left(\nu^{-1}(\lambda-\mu)-s_{0}\right) e^{-\nu t}, \zeta(t)=e^{-\nu t}\left(\zeta_{0}+w(\psi(t)),\right.
$$

where $\psi(t)=\nu^{-1}(\lambda-\mu)\left(e^{2 t \nu}-1\right)-\nu^{-1}\left(\lambda-\mu-\nu s_{0}\right)\left(e^{t \nu}-1\right)$.

We mention that if $\lambda \geq \mu$ then (4.1) is true for any $T>0$ and in this case we have a quasi-stationary point $s^{*}=\nu^{-1}(\lambda-\mu)$.

If $\lambda<\mu$ then (4.1) is true on each interval $[0, T]$ such that $T \leq \nu^{-1} \ln \left(\left(\mu-\lambda+\nu s_{0}\right) /(\mu-\lambda)\right)$.

\subsection{Semi-Markov models}

Consider semi-Markov type queueing system $S M / M_{S M, Q} / 1 / \infty$. Let $x(t)$, $t \geq 0$ be a $S M P$ with values in $X$ such that the embedded $M P x_{k}, k \geq 0$ is uniformly ergodic with stationary measure $\pi(A), A \in B_{X}$. Denote by $\tau(x)$ a sojourn time in the state $x, x \in X$. Let non-negative functions $\mu(x, \alpha), x \in X, \alpha \geq 0$, be given. There is one server and infinite number of places for waiting. Calls enter the system one at a time in the epochs of jumps $t_{1}<t_{2}<\ldots$ of the process $x(t)$. If a call enters the system at a time $t_{k}$ and the number of calls in the system becomes equal to $Q$, then the intensity of service on the interval $\left[t_{k}, t_{k+1}\right)$ is $\mu\left(x_{k}, n^{-1} Q\right)$.

Let $S_{n 0}$ be the initial number of calls, and $Q_{n}(t)$ denote the number of calls in the system at the time $t$.

We put $\quad m(x)=\mathbf{E} \tau(x), m=\int_{X} m(x) \pi(\mathrm{d} x), b(\alpha)=(1-c(\alpha)) m^{-1}$, $c(\alpha)=\int_{X} \mu(x, \alpha) m(x) \pi(\mathrm{d} x), \quad d^{2}(x)=\operatorname{Var} \tau(x), d^{2}=\int_{X} d^{2}(x) \pi(\mathrm{d} x)$, $e_{1}(\alpha)=\int_{X} \mu^{2}(x, \alpha) d^{2}(x) \pi(\mathrm{d} x), e_{2}(\alpha)=\int_{X} \mu(x, \alpha) d^{2}(x) \pi(\mathrm{d} x)$, $D^{2}(\alpha)=c(\alpha)+e_{1}(\alpha)+2(1-c(\alpha)) m^{-1} e_{2}(\alpha)+\left((1-c(\alpha)) m^{-1}\right)^{2} d^{2}$, $g(x, \alpha)=1-m(x)(1-c(\alpha)+\mu(x, \alpha) m) m^{-1}, G(\alpha)=b^{\prime}(\alpha)$. 
Theorem 4.2. Suppose that functions $\mu(x, \alpha)$ are locally Lipschitz with respect to $\alpha$ uniformly in $x \in X, m>0$, function $c(\alpha)$ has no more then linear growth and $n^{-1} Q_{n}(0) \stackrel{\mathrm{P}}{\longrightarrow} s_{0}$. Then the relation (4.1) holds, where $d s(t)=\left(1-c(s(t)) m^{-1} \mathrm{~d} t, s(0)=s_{0}\right.$ and $T$ is any positive value such that $s(t)>0, t \in[0, T]$.

If in addition the variables $\tau(x)^{2}$ are uniformly in $x$ integrable, function $c(\alpha)$ is continuously differentiable, $n^{-1 / 2}\left(Q_{n}(0)-n s_{0}\right) \stackrel{\text { w }}{\Rightarrow} \gamma_{0}$, and there exists a variable $B^{2}(a)=\mathbf{E}\left(g\left(x_{0}, \alpha\right)^{2}+2 \sum_{k=1}^{\infty} g\left(x_{0}, \alpha\right) g\left(x_{k}, \alpha\right)\right)$, where $\mathbf{P}\left\{x_{0} \in A\right\}=\pi(A), A \in B_{X}$, then the sequence of processes

$\gamma_{n}(t)=n^{-1 / 2}\left(Q_{n}(n t)-n s(t)\right)$ weakly converges in $\mathcal{D}_{T}$ to a diffusion process $\gamma(t)$ such that $\gamma(0)=\gamma_{0}$ and

$$
\mathrm{d} \gamma(t)=G(s(t)) \gamma(t) \mathrm{d} t+m^{-1 / 2}\left(D^{2}(s(t))+B^{2}(s(t))\right)^{1 / 2} \mathrm{~d} w(t) .
$$

Proof. We may represent the process $Q_{n}(t)$ as PSMS. Here times $t_{k}$ are switching times and variable $\xi_{n k}(x, n \alpha)$ can be represented in a form:

$$
\xi_{n 1}(x, n \alpha)=1-\Pi_{\mu(x, \alpha)}(\tau(x)),
$$

where $\Pi_{\lambda}(t)$ is a Poisson process with parameter $\lambda$. It is easy to see that $\mathbf{E} \xi_{1}(x, n \alpha)=1-\mu(x, \alpha) m(x)$ and not so difficult to calculate another characteristics and apply Theorems 3.1,3.2. We mention also that due to the condition $s(t)>0, t \in[0, T]$ and Uniform convergence in (4.1) we get that $\mathbf{P}\left\{Q_{n}(n t)>0, t \in[0, T]\right\} \rightarrow 1$ that is the trajectory of a constructed $R P S M$ is asymptotically equivalent to the trajectory of the queue.

In particular when $\mu(x, \alpha) \equiv \alpha \mu$, our system is equivalent to system $S M / M / \infty$ with semi-Markov input and exponential service. In that case $c(\alpha)=\alpha \mu m, b(\alpha)=1 / m-\alpha \mu, G(\alpha)=-\mu, D(\alpha)^{2}=\mu m \alpha+$ $d^{2} / m^{2}, g(x, \alpha)=1-m(x) / m$, and we obtain $s(t)=(\mu m)^{-1}-\left((\mu m)^{-1}-s_{0}\right) e^{-\mu t}$.

Some other examples of non-Markov and even non-semi-Markov models of the types $G_{Q} / M_{Q} / 1 / \infty, S M_{Q} / M_{Q} / 1 / \infty$ and $\left(G_{Q} / M_{Q} / 1 / \infty\right)^{r}$ are considered by Anisimov (1989, 1995ab).

\subsection{Retrial queueing systems}

In retrial systems customers finding the server busy may join the special retrial queue and repeat their attempts for service after some random time. 
1). System $M_{Q} / G / 1 / w . r$. Consider a system with one server and infinitely many places for waiting. Calls enter the system one at a time. If the server is free it immediately takes the call for service. If the server is busy the call will wait in a queue.

Suppose that system characteristics depend on some parameter $n, n \rightarrow$ $\infty$. Let $\lambda(q), \nu(q), q \geq 0$ be given non-negative functions. Denote by $Q_{n}(t)$ the number of calls in the queue at time $t$. Let $t_{n 1}<t_{n 2}<\ldots$ be sequential epochs of finishing service, $t_{n 0}=0$. Denote $Q_{n k}=Q_{n}\left(t_{n k}+0\right)$. We assume that on the interval $\left[t_{n k}, t_{n k+1}\right)$ an input flow is a Poisson one with parameter $\lambda\left(n^{-1} Q_{n k}\right)$ and each call in the queue independently of other calls with local intensity $n^{-1} \nu\left(n^{-1} Q_{n k}\right)$ may re-apply for service. If the server is free it takes the call for service. If the server is busy the call remains in the queue and repeats its attempts for service later in the same way. A service time $\kappa_{n}$ doesn't depend on that, if a call comes from an input flow or from the queue, and has a general distribution function $B_{n}(x)=\mathbf{P}\left(\kappa_{n} \leq x\right)$ with finite moments of the first and second order $m_{n}$ and $m_{n}^{(2)}$. We denote this system as $M_{Q} / G / 1 / w \cdot r$.

Theorem 4.3. 1). If functions $\lambda(q), \nu(q)$ are locally Lipschitz, $\lambda(q)>$ $0, \nu(q)>0, q \geq 0, \Lambda(q)=\lambda(q)+q \nu(q) \leq L(1+q)$, as $n \rightarrow \infty m_{n} \rightarrow$ $m, n^{-1} Q_{n}(0) \stackrel{\mathrm{P}}{\longrightarrow} s_{0}$, and variables $\kappa_{n}$ are uniformly integrable, then for any $T>0$

$$
\sup _{0 \leq t \leq T}\left|n^{-1} Q_{n}(n t)-s(t)\right| \stackrel{\mathrm{P}}{\longrightarrow} 0 .
$$

Here the function $s(t)$ satisfies the equation:

$$
\mathrm{d} s(t)=\left(\lambda(s(t))-m(s(t))^{-1}\right) \mathrm{d} t,
$$

where $m(q)=m+(\lambda(q)+q \nu(q))^{-1}$.

2.) If in addition to these conditions functions $\lambda(\cdot)$ and $\nu(\cdot)$ are continuously differentiable and as $n \rightarrow \infty$

$$
\sqrt{n}\left(m_{n}-m\right) \rightarrow 0, m_{n}^{(2)} \rightarrow m^{(2)}, n^{-1 / 2}\left(Q_{n}\left(n t-n s_{0}\right) \stackrel{\text { p }}{\Rightarrow} \zeta_{0},\right.
$$

and variables $\kappa_{n}^{2}$ are uniformly integrable, then the sequence of processes $\zeta_{n}(t)=n^{-1 / 2}\left(Q_{n}(n t)-n s(t)\right), t \geq 0$ weakly converges in $\mathcal{D}_{T}$ for any $T>0$ to a diffusion process $\zeta(t)$ :

$$
\mathrm{d} \zeta(t)=g(s(t)) \zeta(t) \mathrm{d} t+m(s(t))^{-1 / 2} D(s(t)) \mathrm{d} w(t), \zeta(0)=\zeta_{0} .
$$


Here

$$
\begin{gathered}
g(q)=\frac{\mathrm{d}}{\mathrm{d} q}\left(\lambda(q)-m(q)^{-1}\right) \\
D^{2}(q)=\lambda(q) m(q)+\sigma(q)^{2} m(q)^{-2}-2 \lambda(q) m(q)^{-1}(\lambda(q)+q \nu(q))^{-2} \\
\sigma^{2}(q)=\sigma^{2}+(\lambda(q)+q \nu(q))^{-2}, \sigma^{2}=m^{(2)}-m^{2}
\end{gathered}
$$

Remark 4.1. As the function $s(t)$ has continuous derivative and for any $t$ such that $s(t)=0$ we get $s^{\prime}(t)=\lambda(0)-\lambda(0)(1+\lambda(0) m)^{-1}>0$, then from the relation $s_{0} \geq 0$ it follows that a solution of the equation (4.3) is positive on any interval $[0, T]$.

Proof. We represent the process $Q_{n}(t)$ as a $S P$. Let us choose times $t_{n k}$ as switching times. Denote $\tau_{n k}(n q)=t_{n k+1}-t_{n k}$ given that $Q_{n k}=n q$. According to the construction $\mathbf{P}\left(\tau_{n k}(n q) \leq x\right)=\mathbf{P}\left(\eta(\Lambda(q))+\kappa_{n} \leq x\right)$ where $\eta(\Lambda(q))$ is an independent of $\kappa_{n}$ exponentially distributed random variable with parameter $\Lambda(q)$. Further denote $\xi_{n k}\left(Q_{n k}\right)=Q_{n k+1}-Q_{n k}$. Then we can write a representation:

$$
\begin{gathered}
\mathbf{P}\left(\xi_{n k}\left(Q_{n k}\right) \leq x / Q_{n k}=n q, \kappa_{n}=z\right)=(\lambda(q)+q \nu(q))^{-1} \times \\
\left(q \nu(q) \mathbf{P}\left(\Pi_{\lambda(q)}(z)-1 \leq x\right)+\lambda(q) \mathbf{P}\left(\Pi_{\lambda(q)}(z) \leq x\right)\right),
\end{gathered}
$$

where we denote by $\Pi_{b}(t)$ a Poisson process with parameter $b$.

Now if we introduce an embedded process $\widehat{Q}_{n}(t)=Q_{n k}$ as $t_{n k} \leq t<$ $t_{n k+1}$, then the process $\widehat{Q}_{n}(t)$ is a $R P S M$ constructed with the help of variables $\tau_{n k}(n q), \xi_{n k}(n q)$. It is not so difficult to calculate first and second moments of these variables and using results of the paper (Anisimov and Aliev, 1990) to prove Theorem 4.3 for the process $Q_{n}(t)$. Further in our case due to monotone behaviour of the trajectory of $Q_{n}(t)$ on each interval $\left[t_{n k}, t_{n k+1}\right)$ and the convergence of $\widehat{Q}_{n}(t)$ conditions 2$)$ of Theorem 3.1 and 1) of Theorem 3.2 are automatically fulfilled. That finally implies the statement of Theorem 4.3 .

In particular if $\lambda(q) \equiv \lambda, \nu(q) \equiv \nu$ we get a classical retrial system. In this case if $\lambda m<1$ there exists a stationary point $s^{*}$ of the equation (4.3): $s(t) \rightarrow s^{*}=\lambda^{2} m((1-\lambda m) \nu)^{-1}$. In stationary case $\left(s_{0}=s^{*}\right)$ we get $s(t) \equiv s^{*}$ and the process $\zeta(t)$ satisfies the equation:

$$
\mathrm{d} \zeta(t)=-(1-\lambda m)^{2} \nu \zeta(t) \mathrm{d} t+\lambda \sqrt{\lambda \sigma^{2}+2 m-\lambda m^{2}} \mathrm{~d} w(t), \zeta(0)=\zeta_{0} .
$$


This is Ornstein-Uhlenbeck process and after solving (4.4) we get:

$$
\zeta(t)=e^{-a t} \zeta_{0}+b \int_{0}^{t} e^{-a(t-u)} \mathrm{d} w(u)
$$

where $a=(1-\lambda m)^{2} \nu, b^{2}=\lambda^{2}\left(\lambda \sigma^{2}+2 m-\lambda m^{2}\right)$.

We remark that as $t \rightarrow \infty$ the distribution of $\zeta(t)$ weakly converges to Gaussian distribution with parameters $\left(0, b^{2}(2 a)^{-1}\right)$. That is in stationary case at large $n$ and $t$ we can use an approximation:

$$
Q_{n}(n t) \approx n s^{*}+\sqrt{n} b(2 a)^{-1 / 2} \mathcal{N}(0,1) \text {. }
$$

The result of Theorem 4.3 is also true if we have dependence of functions $\lambda(\cdot), \nu(\cdot)$ on the current number of calls $Q_{n}(t)$.

These results can be extended on the base of Theorems 3.1, 3.2 on the case when we have additional Markov switches at the epochs $t_{n k}$, and on the case when the server is not reliable (see Anisimov, Atadzhanov, 1994).

We mention that in (Anisimov, 1999) analogous results ( $A P$ and $D A$ ) were obtained for a system $\bar{M}_{Q} / \bar{G} / \overline{1} / w . r$ which is described as a one-server system with multiple Poisson input (a call of a type $i$ has an input rate $\left.\lambda_{i}, i=1, . ., r, r<\infty\right)$, general service depending on the type of a call, and intensities of repeated calls in the queue $\left\{\nu_{i}(\bar{q}), \bar{q} \in \mathcal{R}_{+}^{r}\right\}$ depending on the current vector of all waiting calls.

2). System $M / M / m / w \cdot r$. Consider now a system with $m$ identical servers and intensity of service $\mu$. An input is a Poisson flow of identical calls with parameter $\lambda$. Denote by $R_{n}(t)$ a number of busy servers at time $t$. Let families $\left\{p_{i}(s), q_{i}(s), r_{i}(s), i=0,1, . ., m\right\}$ and $\{\nu(s), \alpha(s), g(s)\}, s \geq 0$ of continuous nonnegative functions be given. Here $p_{i}(s)+q_{i}(s)+r_{i}(s)=1, \alpha(s)+g(s)=1$ for any $s \geq 0, i=0,1, . ., m$.

Let $Q_{n}(t)$ denotes the number of waiting calls at time $t$. Then if a call enters the system at time $t$ and $\left(R_{n}(t), Q_{n}(t)\right)=(i, n q) \quad(i \leq r)$ then with probability $p_{i}(q)$ it immediately takes one of free servers, with probability $q_{i}(q)$ the call directly goes to the queue, and with probability $r_{i}(q)$ the call gets a refusal and leaves the system (if $i=m$ then we put $p_{m}(q) \equiv 0$ ). If $Q_{n}(t)=n q$, each call in the queue independently of others can re-apply for service with local intensity $n^{-1} \nu(q)$. If there is a free server, it immediately takes the call to serve. If a call finds all servers busy, then it remains in the queue with probability $\alpha(q)$ or with probability $g(q)$ it leaves the system. 
The process $\left(R_{n}(t), Q_{n}(t)\right)$ is a $M P$ and we can represent it as a $R P S M$ and using the result of Anisimov $(1991,1992)$ it is possible under some conditions of regularity to prove that the process $n^{-1} Q_{n}(n t)$ weakly converges in $\mathcal{D}_{T}$ to a solution of a differential equation (see Anisimov, 1999).

These results show that a technique based on limit theorems of $A P$ and $D A$ types for $S P$ gives us a new effective approach for study transient and stable regimes of operating rather complex queueing models under overloading conditions. It also gives us a possibility instead of direct simulation to use an approximate relation $Q_{n}(n t) \approx n s(t)+\sqrt{n} \zeta(t)$ for calculation different reliability and cost functionals of the system.

\section{References}

Anisimov, V.V. (1973). Asymptotic consolidation of the states of random processes, Cybernetics, 9, 3, 494-504.

Anisimov, V.V. (1975). Limit theorems for random processes and their application to discrete summation schemes. Theory Probab.Appl., 20, 692-694

Anisimov, V.V. (1977). Switching processes. Cybernetics, 13, 4, 590-595.

Anisimov, V.V., Zakusilo, O.K. and V.S. Dontchenko (1987). The elements of queueing theory and asymptotic analysis of systems, Publ. "Visca Scola", Kiev, 248 p. (Russian).

Anisimov, V.V. (1988). Random Processes with Discrete Component. Limit Theorems. Publ. Kiev Univ., 184 p., (Russian).

Anisimov V.V. (1989). Limit theorems for Switching Processes. Functional Analysis III , Proc. Postgrad. School and Conf., Dubrovnik, Yugoslavia, 1989; Var. publ. ser., Aarhus Univ., 1992, 40, 235-262.

Anisimov, V.V. and A.O. Aliev (1990). Limit theorems for recurrent processes of semi-Markov type. Theor. Probab. and Math. Statist., 41, 7-13.

Anisimov, V.V. and Kh.L. Atadzhanov (1991). Diffusion approximation of systems with repeated calls. Theor. Probab. and Math. Statistics., 44, 3-8.

Anisimov, V.V. (1991). Averaging principle for switching recurrent sequences. Theor. Probab. and Math. Statist., 45, 1-8.

Anisimov, V.V. and E.A. Lebedev (1992) Stochastic Queueing Networks. Markov Models, Publ. Kiev Univ., 206 p. (Russian).

Anisimov, V.V. (1992). Averaging principle for switching processes Theory Probab. and Math. Statist., 46, 1-10.

Anisimov, V.V. (1994). Limit theorems for processes with semi-Markov switchings 
and their applications. Random Operators and Stochastic Equations, VSP, Utrecht, The Netherlands, 2, 4, 333-352.

Anisimov, V.V. and Kh.L. Atadzhanov (1994). Diffusion approximation of systems with repeated calls and unreliable server. Journal of Mathematical Sciences, 72, 3032-3034.

Anisimov, V.V. (1995a). Switching processes: averaging principle, diffusion approximation and applications. Acta Applicandae Mathematicae, Kluwer, The Netherlands, 40, 95-141.

Anisimov, V.V. (1995b). Diffusion approximation in switching stochastic models and applications. Exploring Stochastic Laws, Eds A.V. Skorokhod, Yu.V. Borovskikh, VSP, The Netherlands, pp. 13-40

Anisimov, V.V. (1996). Asymptotic analysis of switching queueing systems in conditions of low and heavy loading. In Matrix-Analytic Methods in Stochastic Models, Lect. Notes in Pure and Appl. Mathem. Series, Marcel Dekker, Inc., 183, 241-260.

Anisimov, V.V. (1998). Asymptotic Analysis of Stochastic Models of Hierarchic Structure and Applications in Queueing Models. in Advances in Matrix Analytic Methods For Stochastic Models, Eds. A. S. Alfa, S. R. Chakravarthy, Notable Publ. Inc., USA.

Anisimov, V.V. (1999). Averaging methods for transient regimes in overloading retrial queuing systems. Mathematical and Computing Modeling, 30, 65-78 .

Artalejo, J.R. (1995). A queueing system with returning customers and waiting line. Operations Research Letters, 17, 191-199.

Artalejo, J.R. and G.I. Falin (1996). On the orbit characteristics of the M/G/1 retrial queue. Naval Research Logistics, 43, 1147-1161.

Basharin, G.P., Bocharov, P.P. and Ja.A. Kogan (1989). Analysis of Queues in Computing Networks, Publ. Nauka, Moscow, (Russian).

Buslenko, N.P., Kalashnikov, V.V. and I.N. Kovalenko (1973). Lectures on the Theory of Complex Systems, Publ. Sov. Radio, Moscow, (Russian).

Chen, H. and A. Mandelbaum (1994). Hierarchical modelling of stochastic networks, Part I, II. In D.D. Yao (ed.), Stochastic Modeling and Analysis of Manufacturing Systems, Springer-Verlag, 47-105; 107-131.

Ez̃ov, I.I. and A.V. Skorokhod (1969). Markov processes which are homogeneous in the second component. Theor. Probab. Appl., 14, 679-692.

Falin, G.I. (1990) A survey of retrial queues. Queueing Systems, 7, 127-168.

Falin, G.I., and J.R. Artalejo (1995). Approximation for multiserver queues with balking/retrial discipline. OR Spektrum, 17, 239-244. 
Falin, G.I. and J.G.C. Templeton (1997). Retrial Queues, Chapman and Hall.

Gikhman, I.I. and A.V. Skorokhod (1973). Theory of Random Processes, II, Publ. "Nauka", Moscow, 640 p. (Russian).

Harrison, J.M. (1995). Balanced fluid models of multiclass queueing network: a heavy traffic conjecture. Stochastic Networks, IMA Volumes in Math. and its Appl., Eds. F. Kelly and R. Williams, Springer-Verlag, New York, 71, 1-20.

Harrison, J.M. and R.J. Williams (1996). A multiclass closed queueing network with unconventional heavy traffic behavior, Ann. Appl. Probab., 6, 1, 1-47.

Hersh, R. (1974). Random evolutions: survey of results and problems. Rocky Mount. J. Math., 4, 3, 443-475.

Kertz, R. (1978). Random evolutions with underlying semi-Markov processes, Publ. Res. Inst. Math. Sci., 14, 589-614.

Khas'minskii, R.Z. (1968). About the averaging principle for ITO stochastic differential equations. Kybernetika, 4, 3, 260-279

Kulkarni, V.G. and H.M. Liang (1997). Retrial queues revisited. In: Frontiers in Queueing. Models and Applications in Science and Engineering, Edt. J.H. Dshalalow, CRC Press, 19-34.

Koroljuk, V.S. and A. V. Swishchuk (1994). Random Evolutions, Kluwer Ac. P.

Kurtz, T. (1973). A limit theorem for perturbed operator semigroups with applications to random evolutions. J. Funct. Anal, 12, 55-67.

Mandelbaum, A. and G. Pats (1998). State-dependent stochastic networks. Part I: Approximations and applications with continuous diffusion limits. Ann. Appl. Prob., 8, 2, 569-646.

Martin, M. and J.R. Artalejo (1995). Analysis of an M/G/1 queue with two types of impatient units. Advances in Applied Probability, 27, 840-861.

Papanicolaou, G. and R. Hersh (1972). Some limit theorems for stochastic equations and applications. Indiana Univ. Math. J., 21, 815-840.

Pinsky, M. (1975a). Multiplicative operator functionals and their asymptotical properties. Adv. in Prob., 3, 1-100.

Pinsky, M. (1975b). Random evolutions. Lecture Notes in Math., 451, Springer, New York, 89- 100.

Skorokhod, A.V. (1989). Asymptotic Methods in the Theory of Stochastic Differential Equations. Amer. Math. Soc., Providence, RI.

Yang, T. and J.G.C. Templeton (1987). A survey on retrial queues. Queueing Systems, 2, 203-233. 